\title{
Innovative treatment as a precursor to clinical research
}

\author{
David Wendler, ${ }^{1}$ Seher Anjum, ${ }^{2}$ and Peter Williamson ${ }^{2}$
}

'Department of Bioethics, NIH Clinical Center, Bethesda, Maryland, USA. ²aboratory of Clinical Immunology \& Microbiology, National Institute of Allergy and Infectious Diseases, Bethesda, Maryland, USA.

$\mathbf{R}$ eliance on randomized controlled trials (RCTs) as the gold standard for assessing therapeutics began in 1948, with the widely cited trial of streptomycin for pulmonary tuberculosis (1). Less widely recognized is the fact that the trial was preceded by a retrospective analysis of 92 cases of innovative treatment for miliary and meningeal tuberculosis (2). Since then, the use of innovative treatments prior to formal trials has fallen out of favor and is now actively discouraged.

"Innovative" treatments are treatments that "depart in a significant way from standard or accepted practice" (3). The use of innovative treatments is common in many areas of medicine, including surgery, reproductive medicine, and oncology $(4,5)$. For example, it has been estimated that the majority of advances in surgery are the result of innovation, not clinical trials (6). At the same time, the use of innovative treatments raises important ethical challenges. By deviating from standard care, innovative treatments can pose significant risks and offer uncertain benefits. Unchecked, innovative practice can lead to the dissemination of ineffective, even harmful, interventions.

In response to these concerns, commentators argue that innovative treatments should be used no more than a few times before they are tested in formal clinical trials, at which point the interventions found safe and effective can be offered to patients (7). To enforce this approach, some institutions place a numerical cap on the number of patients, often as few as 3, who may be treated with innovative treatments before they are subjected to clinical trials $(8,9)$. This approach, which has become the standard paradigm for the development of new interventions, ensures that they undergo rigorous testing before being offered to patients and disseminated widely.
Yet, initiating formal trials can take time, sometimes years, resulting in lost opportunities for patients who need treatment urgently (10) and frequently involves randomizing some patients to a no-treatment control group. Mandating that new interventions are evaluated in formal trials, before clinicians have enough experience using them, can also make it difficult to determine which version to test. Which dose? What dosing schedule? Preclinical testing in the laboratory and in animals, along with phase I trials, sometimes provides sufficient information to answer these questions. In other cases, the standard paradigm can lead to premature testing, wasted resources, and the rejection of interventions that would have been found safe and effective if administered in a different way: "Outcomes from trials begun too soon after the introduction of a procedure may reflect a lack of sufficient experience with the new technique and not measure efficacy of the procedure when done after the learning curve flattens" (11). For example, experience revealed that a simple change in the dose of corticosteroids was much more effective at reducing mortality in COVID-19 infections $(12,13)$. And it has been argued that the majority of effective surgical procedures would have been rejected if they had been subjected to formal clinical trials before surgeons had enough experience to determine how to perform them (14).

These observations suggest that, rather than representing a historical anomaly, the use of innovative treatments prior to the streptomycin trial represents an important and appropriate exception to the now standard paradigm for developing new therapeutics. When clinicians do not know how best to deliver a new intervention, they should be permitted to offer it to patients for whom standard interventions are unavailable or ineffective. Patients

Conflict of interest: SA and PW receive research funding from Matinas Biopharma.

who need urgent care can thereby access potential treatments, while clinicians gain experience providing them. Once the clinicians identify an approach to providing the treatment that works for their patients, the identified approach can be tested in formal trials to determine whether it is effective for patients generally. The value of permitting such exceptions to the standard paradigm is illustrated by a recent example.

\section{A new protocol for treating cryptococcal meningoencephalitis}

Cryptococcal meningoencephalitis is a principal cause of nonviral meningitis and is increasingly diagnosed in otherwise healthy individuals. Effective therapy remains elusive, and mortality is estimated at $30 \%$ to $50 \%$ (15). Moreover, those who survive often experience significant disability.

Previous efforts to improve treatment focused on identifying better methods for pathogen killing. More recent efforts have added attention to the inflammatory sequelae. In particular, the optimal timing of initiation, duration, and dose of corticosteroids is unknown in otherwise healthy individuals. Researchers thus treated a series of patients with innovative therapy, trying to do what was best for the specific individuals.

The initial patients were treated with moderate doses of steroids (prednisone, $1 \mathrm{mg} / \mathrm{kg} / \mathrm{d}$ ), which resulted in modest responses, at best, over 1 month. In addition, a cerebrospinal fluid inflammatory rebound accompanied by clinical deterioration was observed in several patients. The protocol was thus adjusted in several stages for subsequent patients, eventually leading to a regimen of intravenous methylprednisolone $1 \mathrm{~g}$ daily for 1 week, followed by $1 \mathrm{mg} / \mathrm{kg} / \mathrm{d}$ oral prednisone, which is tapered based on clinical and radiological response, plus oral fluconazole. In 15 patients who had experienced deterioration on standard therapy over a median period of 6 weeks, this regimen was associated with rapid clinical improve- 


\section{Table 1. Guidelines for innovative practice that precedes formal testing}

\begin{tabular}{|c|c|c|}
\hline Guideline & Explanation & Criteria \\
\hline Independent review & Review and approval by independent clinician(s) & $\begin{array}{l}\text { 1. Sufficient reason to think might benefit the patient } \\
\text { 2. Risks acceptable given nature of the patient's condition } \\
\text { 3. No clearly superior alternative(s) } \\
\text { 4. Consider ways to improve proposed treatment } \\
\text { 5. Ongoing oversight }\end{array}$ \\
\hline Enhanced consent & Ensure patient understands & $\begin{array}{l}\text { 1. Disclose intervention not validated } \\
\text { 2. Disclose all risks, including potential unknown ones } \\
\text { 3. Disclose uncertainty of benefit } \\
\text { 4. Confirm patient understanding } \\
\text { 5. Consider need for independent consent monitor }\end{array}$ \\
\hline Appropriate before research & Assess whether better to conduct trial & $\begin{array}{l}\text { 1. Insufficient information on how to provide } \\
\text { 2. Need to learn before testing } \\
\text { 3. Provision will help to identify protocol for testing } \\
\text { 4. Appropriate documentation and record keeping }\end{array}$ \\
\hline Outcome reporting & Document and report outcomes & $\begin{array}{l}\text { 1. Carefully monitor patients } \\
\text { 2. Record outcomes } \\
\text { 3. Report outcomes to independent reviewer(s) }\end{array}$ \\
\hline
\end{tabular}

ment (16). This finding suggests that the identified regimen could provide the basis for clinical trials to evaluate whether it is effective for the general population of otherwise healthy individuals with cryptococcal meningitis.

When it is unknown how to implement a new intervention, this case illustrates the value of permitting clinicians to provide it as innovative therapy first. This approach provides access to potential treatments for patients who have no known effective treatment options. It also provides clinicians with the experience necessary to identify a regimen that works in their patients, which can then be formally tested in the general patient population. This example thus suggests that we should reconsider the relationship between clinical care and clinical research. In some cases, clinical care in the form of innovative treatments should be the precursor to, and provide a basis for, clinical trials.

\section{Potential objections}

Innovative therapy is research. The present proposal endorses clinicians offering innovative interventions to their patients, and potentially learning from the experience. One might thus object that this approach involves the use of experimental interventions that is covered by research regulations, including review and approval by an institutional review board.
The use of innovative treatments is experimental in the sense that it poses unknown risks and offers uncertain benefits. But, it does not follow that the use of innovative treatments is experimental in the sense of constituting research (17) Whether an activity constitutes research depends on whether it collects generalizable knowledge (3). Generalizable knowledge involves information that applies to a general patient population as opposed to a nonrepresentative subset of the population. This explains why quality improvement efforts that collect information on how to improve care within a specific institution do not constitute research, even though they involve systematic attempts to improve clinical care $(18,19)$.

The example of quality improvement efforts that do not constitute research highlights several considerations that can help to draw the line between the learning from experience that occurs in essentially all clinical care, including innovative treatment, and the conduct of research. First, the provision of clinical care involves accepting whichever patients present. Conducting research involves actively seeking patients who are representative of the population with the condition under study and treating only those who satisfy formal inclusion/exclusion criteria.

Second, clinicians tailor treatments to what they think is best for the present patient. Researchers administer treatments using a fixed protocol that is based on the characteristics of the general patient population.

Third, the learning that takes place during clinical care involves determining which version of a treatment is best for the patients one happens to see. The learning that takes place in research involves formally testing a priori hypotheses with the goal of collecting generalizable knowledge about the patient population.

In some cases, clinicians may alter an innovative therapy with an eye to determining what works best for the general patient population. For example, they might treat their patients based on a fixed regimen that they think makes sense for the general patient population, or they might actively seek patients who are representative of that population. In these cases, provision of innovative therapy may itself constitute research. In contrast, when clinicians accept whichever patients present and treat them in the way that is best for the individual, without attempting to collect generalizable knowledge, the use of innovative therapy does not constitute research, even though clinicians frequently learn from the experience.

Departing from standard care without prior formal testing can expose patients to excessive risks. To protect them, critics might argue that innovative therapy should 
be treated as though it involves research. Although this approach offers patients some protection, it also has the potential to force innovative practice to satisfy inappropriate standards that can undermine the interests of the present patients (10). For example, this approach might force clinicians to develop a formal treatment protocol rather than tailoring treatment to the individual patient. It might also force clinicians to develop explicit inclusion/ exclusion criteria and reject patients who do not satisfy them. A better approach for protecting patients is to develop guidelines and a review process specific to innovative practice (Table 1 and refs. 20-23).

Treating patients prior to research can disturb equipoise. Gaining experience with a new intervention prior to testing it formally may result in clinicians accumulating evidence that the intervention is superior to existing treatments. Prior use as innovative therapy may thus disturb equipoise, raising concern regarding the ethics of randomizing patients in subsequent clinical trials.

This concern, while important, is not unique to the present proposal. The standard paradigm involves conducting in vitro, phase I and II trials prior to randomized phase III trials. These steps can also yield evidence that the treatment being tested is superior to existing treatments. Nonetheless, it can still be ethical to conduct randomized trials as long as they have the potential to collect socially valuable data and they do not expose participants to excessive risks (24).

\section{Summary}

The standard paradigm of testing new interventions in clinical trials before they are offered clinically has the potential, in some cases, to lead to premature testing, and possibly premature rejection of promising interventions. To address this concern, we have argued that the standard paradigm should be regarded as the default, with exceptions permitted in which clinicians first provide new interventions as innovative therapy. The approach found useful in this setting can then be tested in formal trials to determine whether it is effective for the general patient population. The value of permitting such excep- tions suggests that we should reconsider the relationship between clinical care and clinical research. In limited cases, when it is unknown how to administer an intervention, clinical care in the form of innovative treatment should be the precursor to, and provide a basis for, formal clinical trials.

\section{Acknowledgments}

Thanks to John Nichols, Reidar Lie, and Christine Grady for their comments on earlier drafts of the manuscript. This work was funded by the Intramural Research Programs at the NIH Clinical Center and the National Institute of Allergy and Infectious Diseases (grants AI001123 and AO001124). The views expressed are the authors' own. They do not represent the position or policy of the NIH, DHHS, or the US government.

Address correspondence to: David Wendler, Department of Bioethics, NIH Clinical Center, Bldg. 10, Room 1C118, Bethesda, Maryland 20892-1156, USA. Phone: 301.435.8726; Email: dwendler@nih.gov.

1. Crofton J. The MRC randomized trial of streptomycin and its legacy: a view from the clinical front line. JR Soc Med. 2006;99(10):531-534.

2. [No authors listed]. Streptomycin treatment of tuberculous meningitis. Lancet. 1948;1(6503):582-596.

3. United States. The Belmont Report: Ethical Principles and Guidelines for the Protection of Human Subjects of Research. CreateSpace Independent Publishing Platform; 1978.

4. Barkun JS, et al. Surgical innovation and evaluation 1: evaluation and stages of surgical innovations. Lancet. 2009;374(9695):1089-1096.

5. Dondorp W, de Wert G. Innovative reproductive technologies: risks and responsibilities. Hum Reprod. 2011;26(7):1604-1608.

6. Frader JE, Caniano DA. Research and innovation in surgery, in McCollough LB, et al., eds. Surgical Ethics. Oxford University Press; 1998:216-241.

7. Prasad V. Why randomized controlled trials are needed to accept new practices: 2 medical worldviews. Mayo Clin Proc. 2013;88(10):1046-1050.

8. Partners Human Research Committee. Innovative therapy and diagnosis. https://www. partners.org/Assets/Documents/MedicalResearch/Clinical-Research/InnovativeTherapy-and-Diagnosis.pdf. Accessed June 22, 2021.

9. Stanford University Medical Center. Innovative care guidelines. https://stanfordhealthcare.org/ content/dam/SHC/health-care-professionals/ medical-staff/policies/innovative-careguidelines-final-3-18-11-copy.pdf. Updated March 18, 2011. Accessed June 22, 2021.
10. Earl J. Innovative practice, clinical research, and the ethical advancement of medicine. Am J Bioeth. 2019;19(6):7-18.

11. Frader JE, Flanagan-Klygis E. Innovation and research in pediatric surgery. Semin Pediatr Surg. 2001;10(4):198-203.

12. The RECOVERY Collaborative Group. Dexamethasone in hospitalized patients with Covid-19 - preliminary report. $N$ Engl J Med. 2021;384(8):693-704

13. Monreal E, et al. High versus standard doses of corticosteroids in severe COVID-19: a retrospective cohort study. Eur JClin Microbiol Infect Dis. 2020;40(4):761-769.

14. Bonchek LI. Sounding board. Are randomized trials appropriate for evaluating new operations? NEngl J Med. 1979;301(1):44-45.

15. Adams P. How to stop crypto, a deadly disease so neglected it's missed on the 'neglected' list. Newsweek Magazine. January 4, 2016. Accessed June 22, 2021. https://www.newsweek.com/ crushing-crypto-world-health-organizationneglected-disease- 411193

16. Anjum S, et al. Outcomes in previously healthy cryptococcal meningoencephalitis patients treated with pulse - taper corticosteroids for post-infectious inflammatory syndrome [published online December 31, 2020]. Clin Infect Dis. https://doi.org/10.1093/cid/ciaa1901.

17. Fost N. Ethical dilemmas in medical innovation and research: distinguishing experimentation from practice. Semin Perinatol. 1998;22(3):223-232.

18. Duke University Health System. Human Research Protection Program Quality Improvement Activities in Health Care Versus Research. https:/irb. duhs.duke.edu/sites/irb.duhs.duke.edu/files/ QI\%20policy\%20and\%20checklist.pdf. Updated March 3, 2016. Accessed June 22, 2021.

19. Agency for Healthcare Research Quality. 2012 Annual Progress Report to Congress National Strategy for Quality Improvement in Health Care. U.S. Department of Health and Human Services; 2012.

20. Biffl WL, et al. Responsible development and application of surgical innovations: a position statement of the Society of University Surgeons. JAm Coll Surg. 2008;206(6):1204-1209.

21. American College of Obstetricians Gynecologists. Innovative practice: ethical guidelines. Obstet Gynecol. 2006;108(6):1589-1595.

22. International Society for Stem Cell Research. Guidelines for Stem Cell Research and Clinical Translation. Updated May 12, 2016. Accessed June 22, 2021. https://www. isscr.org/docs/default-source/all-isscrguidelines/guidelines-2016/isscr-guidelinesfor-stem-cell-research-and-clinical-translationd67119731dff6ddbb37cff0000940c19.pdf.

23. Taylor PL. Overseeing innovative therapy without mistaking it for research: a function-based model based on old truths, new capacities, and lessons from stem cells. J Law Med Ethics. 2010;38(2):286-302.

24. Miller FG, Brody H. A critique of clinical equipoise. Therapeutic misconception in the ethics of clinical trials. Hastings Cent Rep. 2003;33(3):19-28. 